\title{
Scalability of the Channel Capacity in Graphene-enabled Wireless Communications to the Nanoscale
}

\author{
I. Llatser, A. Cabellos-Aparicio, E. Alarcón, J. M. Jornet, A. Mestres, H. Lee, and J. Solé-Pareta
}

\begin{abstract}
Graphene is a promising material which has been proposed to build graphene plasmonic miniaturized antennas, or graphennas, which show excellent conditions for the propagation of Surface Plasmon Polariton (SPP) waves in the terahertz band. Due to their small size of just a few $\mu \mathrm{m}$, graphennas allow the implementation of wireless communications among nanosystems, leading to a novel paradigm known as Grapheneenabled Wireless Communications (GWC). In this paper, an analytical framework is developed in order to evaluate how the channel capacity of a GWC system scales as its dimensions shrink. In particular, we study how the unique propagation of SPP waves in graphennas will impact the channel capacity. Next, we further compare these results with respect to the case when metallic antennas are used, in which these plasmonic effects do not appear. In addition, asymptotic expressions for the channel capacity are derived in the limit when the system dimensions tend to zero. In this scenario, necessary conditions to ensure the feasibility of GWC networks are found. Finally, using these conditions, new guidelines are derived to explore the scalability of various parameters, such as transmission range and transmitted power. These results may be helpful for designers of future GWC systems and networks.
\end{abstract}

Index Terms-Scalability, channel capacity, nanonetworks, graphene-enabled wireless communications, graphennas.

\section{INTRODUCTION}

D ESPITE the huge progress of nanotechnology in the last decade, nanosystems (such as nanosensors and nanoactuators) have intrinsic limitations due to their small size, such as their simplicity requirement and short operation range. Communication among nanosystems will allow them to cover larger areas and fulfill more complex tasks by means of information sharing and cooperation. The resulting nanonetworks [1], networks of nanosystems, will boost the range of applications of nanotechnology, bringing new opportunities in fields as diverse as Information and Communication Technologies (ICT) (e.g., Wireless Nanosensor Networks [2], Wireless Networks-on-Chip [3] and the Internet of Nanothings [4]), biomedical technology (e.g., health monitoring and cooperative drug delivery systems [5]), or environmental research (e.g., distributed air pollution control [6]).

I. Llatser, A. Cabellos-Aparicio, E. Alarcón, J. M. Jornet, A. Mestres and J. Solé-Pareta are with the NaNoNetworking Center in Catalunya (N3Cat), Universitat Politècnica de Catalunya, Barcelona, Spain. E-mail: \{llatser, acabello, jmjornet, amestres, pareta $@$ ac.upc.edu, eduard.alarcon@upc.edu

J. M. Jornet is with the Department of Electrical Engineering, University at Buffalo, The State University of New York, Buffalo, NY 14260, USA. E-mail: jmjornet@buffalo.edu

H. Lee is with the Samsung Advanced Institute of Technology (SAIT), South Korea. E-mail: heekwan.lee@samsung.com
However, a nanonetwork is not a mere downscaled version of a conventional network; on the contrary, classical communication paradigms need to undergo a profound revision before being applied to this new scenario. A novel paradigm has recently emerged to implement nanonetworks: Graphene-enabled Wireless Communications (GWC) [2], [3]. This approach is motivated by the doubts about the feasibility of scaling down current metallic antennas, mainly because their resonant frequency would be extremely high [7]. For the expected size of a nanosystem (a few $\mu \mathrm{m})$ [2], the frequency radiated by its antenna would be in the optical range (hundreds of $\mathrm{THz}$ ), resulting in a huge channel attenuation that might render wireless communication at the nanoscale unfeasible.

In order to overcome this limitation, graphene plasmonic miniaturized antennas, or graphennas [7], [8], have been proposed to implement wireless communications among nanosystems. Graphene [9] presents very good conditions for the propagation of Surface Plasmon Polariton (SPP) waves which, as further explained in Sec. II, allow graphennas with a size of a few $\mu \mathrm{m}$ to resonate in the terahertz band $(0.1-10 \mathrm{THz})$ [7], [8], at a frequency up to two orders of magnitude lower than metallic antennas with the same size. These unique plasmonic effects make graphennas a promising candidate to implement wireless communications among nanosystems.

Even though graphennas have only been analyzed to date from a theoretical perspective and by simulation [7], [10], [11], terahertz plasmonic resonances of graphene devices have recently been experimentally observed by several groups [12], [13], [14], [15]. Moreover, prototypes of several graphene devices have been experimentally fabricated and measured in the last few years, such as graphene FET transistors working at frequencies of hundreds of $\mathrm{GHz}$ [16], [17], [18], as well as LNAs, mixers and frequency multipliers [19], [20], [21]. Even complete graphene integrated circuits have been recently demonstrated [22] and have been the object of several patents [23], [24].

Of particular interest is the recent developments in metalgraphene-metal photodectectors, which have been experimentally demonstrated at data rates up to tens of Gbits/s [25], [26]. Due to these achievements, we expect that graphennas will be successfully manufactured in the near future. Since graphennas are envisaged to provide wireless communication capabilities to future nanosystems, an analytical framework that provides guidelines and predicts the performance of GWC will prove useful for designers of graphennas and nanosystems.

In this paper, we analyze the scalability of the channel 


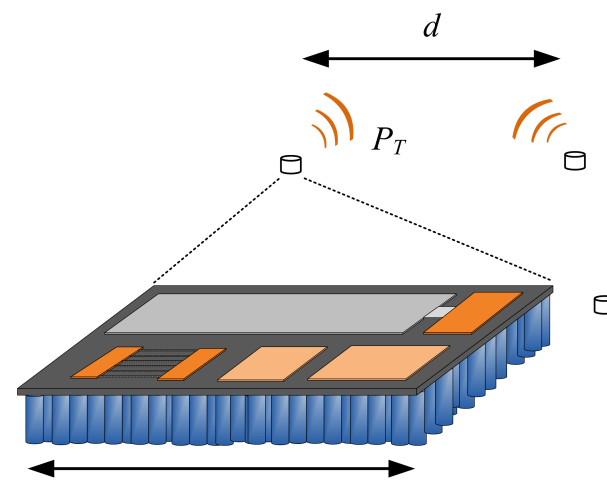

$\Delta$

Fig. 1. Schematic diagram of a nanonetwork implemented by means of GWC (right) and a magnified individual nanosystem (left). The scale parameters, namely the antenna length $\Delta$, the transmission distance $d$ and the transmitted power $P_{T}$, are shown.

capacity in a scenario of GWC, such as the one shown in Fig. 1. Scalability theories have set a framework for the development of circuits based on CMOS technology and derived a roadmap allowing device designers to learn how to make circuits smaller such that the desired performance metrics can be optimized [27], [28], [29]. Inspired by these theories, we investigate the case in which the system size shrinks to the nanoscale. This is opposed to the existing solutions on network scalability in the literature, which consider scenarios in which the network size increases [30], [31], [32]. Our final objective is to derive guidelines that will help researchers to design future GWC networks. We summarize the main contributions of our work as follows:

1. We derive analytical expressions for the scalability of the channel capacity of wireless communications towards the nanoscale as a function of three key scale parameters: the antenna length $\Delta$, the transmission distance $d$ and the transmitted power $P_{T}$ (see Fig. 1). We compare the case in which the radiating element is a graphenna with respect to that of a metallic antenna. Our results show that there are important differences, giving graphennas a scalability advantage over metallic antennas.

2. Based on the previous analytical expressions, we obtain quantitative results of the capacity expressions by assuming numerical values for the parameters, considering a realistic scenario of GWC.

3. We derive guidelines which indicate how the antenna transmission distance and the transmitted power need to scale as a function of the antenna length in order to keep the network feasible. These results establish a general framework which may serve as a guide for designers of future nanonetworks using GWC.

The remainder of this paper is organized as follows. In Sec. II, we outline some of the novel characteristics of GWC with respect to current wireless communications and we obtain analytical expressions and quantitative results of the channel capacity as a function of the scale parameters. Then, in Sec. III, we compute the limit of the obtained channel capacity expressions when the system size shrinks to the nanoscale. In
Sec. IV, we derive scalability guidelines for several network parameters. Finally, Sec. V concludes the paper.

\section{Channel CApacity of GRAPHene-EnABled WIRELESS COMMUNICATIONS}

GWC exhibit several important differences with respect to current wireless communications techniques. We describe next three of the most relevant features of GWC. First, we examine the propagation of SPP waves in graphennas, which cause them to resonate in the terahertz band. Second, we describe the effects of molecular absorption and noise, two novel characteristics of the wireless channel in the terahertz band. Finally, the potential impact of the huge available bandwidth in GWC is discussed.

The propagation speed of EM waves in commonly-used metallic antennas is related to the speed of light $c$. For instance, in resonant metallic patch antennas the propagation speed is $v_{p}=c / \sqrt{\varepsilon_{r}}$, where $\varepsilon_{r}$ is the dielectric constant of the substrate, and the antenna length is typically around half a wavelength: $\Delta \approx \lambda / 2$. As a consequence, the resonant frequency of such an antenna, given by $f_{R}=v_{p} / \lambda \approx c /\left(2 \sqrt{\varepsilon_{r}} \Delta\right)$, is inversely proportional to its length. This relationship imposes a limit in the minimum size of an antenna whose resonance frequency lies in the EM domain (i.e., up to the terahertz band), in the order of $100 \mu \mathrm{m}$, which prevents the possibility of integrating them into nanosystems just a few $\mu \mathrm{m}$ in size.

In order to overcome this issue, nanomaterials such as graphene offer the possibility of building much smaller antennas due to their novel plasmonic properties. Indeed, graphene has been shown to support the propagation of SPP waves at frequencies as low as the terahertz band, both by means of simulation and experimental measurements [8], [10], [12], [33]. The edge of a graphene patch acts as a mirror and it behaves as a Fabry-Perot resonator for transverse-magnetic SPP waves with a unique dispersion relation [34].

In particular, we demonstrated in our previous work [35] that the dispersion relation of SPP waves in graphene causes graphennas to have not only a much lower resonant frequency than their metallic counterparts, but their resonant frequency also scales better as the antenna size is reduced. In particular, the resonant frequency of gold antennas is inversely proportional to their length: $f_{R m}=\Theta(1 / \Delta)$, whereas the resonant frequency of graphennas scales inversely proportional to the square root of the antenna length: $f_{R g}=\Theta(1 / \sqrt{\Delta})$. In consequence, graphennas just a few $\mu \mathrm{m}$ long resonate in the terahertz band, at a much lower frequency than metallic antennas with the same size.

Throughout this paper, we use the subindex $m$ to denote the parameters corresponding to a metallic antenna, and the subindex $g$ for the case in which a graphenna is considered.

Furthermore, graphennas have been also modeled with a tight-binding model and their resonant frequency has been found to scale similarly with respect to the antenna dimensions [7], which validates the trend observed from the analysis of the plasmonic effects in graphennas.

Molecular absorption is the process by which part of the wave energy is converted into internal kinetic energy of the excited molecules in the medium [36]. Molecular absorption acts 


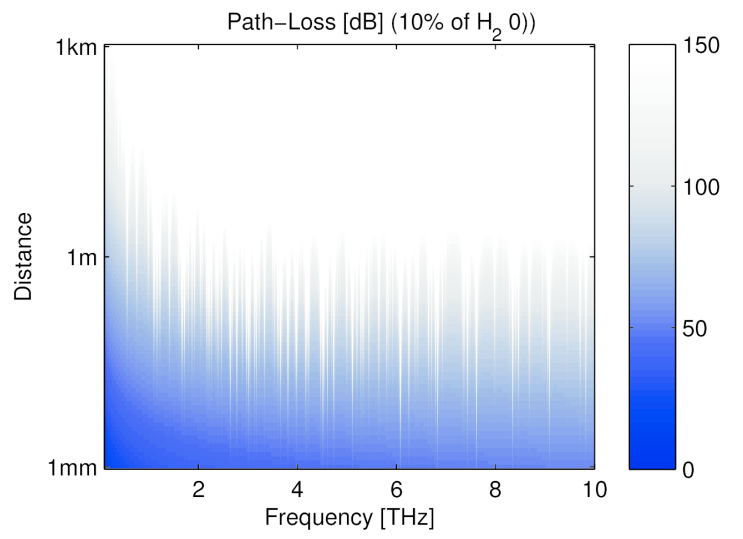

Fig. 2. Total path loss in $\mathrm{dB}$ as a function of frequency and distance in a standard medium with $10 \%$ of water vapor molecules.

as an additional factor of attenuation to the propagated signal in wireless communications in the terahertz band. Fig. 2 [37] shows the total path loss in the terahertz channel, comprising both the free-space path loss and molecular absorption, as a function of frequency and distance.

In addition, the absorption from molecules present in the medium not only attenuates the transmitted signal, but it also introduces noise. Indeed, when a terahertz wave is propagating through the atmosphere, the internal vibration of the molecules in the medium turns into the emission of EM radiation of the same frequency that the incident waves that provoked this motion. Thus, molecular noise needs to be taken into account as a new source of noise in a scenario of GWC.

Since a graphenna is able to radiate EM waves in the terahertz band, the corresponding bandwidth associated to this frequency and, ultimately, the capacity of the GWC will be extremely high (up to Tbits/s). One may think that such a large capacity will not be needed, since it is envisaged that a single nanosystem will have low throughput requirements. However, network designers may take advantage of this large channel capacity in order to design novel protocols specifically adapted to nanonetworks.

In current wireless networks, the channel capacity is usually the bottleneck that limits the network throughput. As a consequence, many networking protocols are designed to require a low bandwidth in order to maximize the network throughput. In contrast, the envisaged huge channel capacity in GWC opens the door to new protocols that take advantage of a virtually unlimited available bandwidth to optimize other performance metrics which may be more relevant in nanonetworks, such as reducing the energy consumption or the complexity of the nanosystems.

Our next goal is to find analytical expressions for the scalability of the channel capacity of wireless communications towards the nanoscale, as a function of three key scale parameters: (i) the antenna length $\Delta$, (ii) the transmission distance $d$, and (iii) the total transmitted power $P_{T}$. We perform this analysis for two scenarios: (i) when the transmitters use metallic antennas to radiate the EM signal, and (ii) when graphennas are used (with their unique features introduced in Section II). The first scenario is taken as a reference in order
TABLE I

PARAMETERS AND CONSTANTS

\begin{tabular}{cl}
\hline \hline Symbol & Quantity \\
\hline$\Delta$ & Antenna length \\
$d$ & Transmission distance \\
$P_{T}$ & Total power radiated by the transmitter \\
$f_{R}$ & Antenna resonant frequency \\
$B$ & Total system bandwidth \\
$c$ & Speed of light in the vacuum \\
$S$ & Power spectral density of the transmitted signal \\
$A$ & Total channel attenuation \\
$A_{s p r e a d}$ & Spreading loss \\
$A_{a b s}$ & Molecular absorption loss \\
$\tau$ & Transmittance of the medium \\
$k$ & Medium absorption coefficient \\
$N$ & Power spectral density of noise at the receiver \\
$k_{B}$ & Boltzmann constant \\
$T_{\text {sys }}$ & System temperature \\
$T_{m o l}$ & Molecular noise temperature \\
$T_{0}$ & Standard temperature \\
$\varepsilon$ & Emissivity of the medium
\end{tabular}

to evaluate the results obtained in the latter case. Interference among several simultaneous transmissions is not considered in this work.

As we previously outlined, a graphenna is able to radiate EM waves in the terahertz band. In consequence, the physical channel in GWC in the very short range potentially allows the transmission of signals with a very large bandwidth [37] with a highly frequency-dependent behavior. In order to compute its capacity, we need to divide the channel in narrow subbands so that, in each sub-band, the channel attenuation and the noise power spectral density can be considered locally flat. The total channel capacity is then obtained as the combined capacity of all its sub-bands. The capacity of a single subband $C_{i}$ is expressed using the Shannon limit theorem [38]:

$$
C_{i}=B_{i} \log _{2}\left(1+\frac{S_{i}}{A_{i} N_{i}}\right)
$$

where $B_{i}$ is the width of the frequency band, $S_{i}$ the power radiated by the transmitter in this band, $A_{i}$ the sub-band attenuation and $N_{i}$ the noise power in the frequency band.

The total channel capacity $C$ can be obtained by taking the limit of the sub-band capacity when $B_{i} \rightarrow 0$ and integrating it over the whole frequency band, which leads to the following expression [39]:

$$
C=\max _{S(f): \int_{B} S(f) d f \leq P_{T}} \int_{B} \log _{2}\left(1+\frac{S(f)}{A(f) N(f)}\right) d f
$$

where $S(f)$ is the power spectral density of the transmitted signal, $A(f)$ stands for the channel attenuation, $N(f)$ is the noise power spectral density at the receiver and $B$ is the system bandwidth. Note that (2) also applies when the noise is not white but frequency-dependent, such as molecular noise.

In order to find an expression for the channel capacity $C$ as a function of the scale parameters, we first concentrate on the magnitudes that determine the channel capacity according to (2): the power spectral density of the transmitted signal $S(f)$, the channel attenuation $A(f)$, the noise power spectral density $N(f)$ and the system bandwidth $B$. Next, we express 
each of them as a function of the scale parameters $\Delta, d$ and $P_{T}$, for the two previously mentioned scenarios, considering a graphenna and a metallic antenna, respectively. Finally, we combine these results in order to find an analytical expression for the channel capacity $C$.

\section{A. Power Spectral Density of the Transmitted Signal}

We next derive an expression for the spectral density of the radiated power by the transmitting antenna. Assuming that the transmitter had a full knowledge of the wireless channel, the optimal power allocation would be achieved through waterfilling. However, since that knowledge would require a feedback loop to send this information from the receiver back to the transmitter, we assume conservatively that the transmitter does not have any knowledge of the channel. In this case, the optimal modulation is known as flash signaling, an asymptotic form of on-off signaling where the on level has unbounded power and the duty cycle is vanishingly small [40]. In this case, the input signal has a flat power spectral density over the entire frequency band:

$$
S(f)= \begin{cases}P_{T} / B & \text { if } 0<f<B \\ 0 & \text { otherwise. }\end{cases}
$$

where $P_{T}$ is the total power radiated by the transmitting antenna.

A modulation based on very short Gaussian pulses specially suited for GWC has recently been proposed [41]. This pulsebased scheme is a promising candidate to modulate the EM waves radiated by graphennas because of the simplicity to generate and detect pulses (there is no carrier) and its high spectral efficiency (a very short Gaussian pulse virtually occupies the whole frequency band). This approach actually constitutes a realistic implementation of flash signaling for GWC and it is accurately approximated by the previous model.

\section{B. Channel Attenuation}

Some of the most promising applications of GWC include Wireless Nanosensor Networks and Wireless Networks-onChip. Wireless Nanosensor Networks involve wireless communications among miniaturized sensors with transmission distances up to $10 \mathrm{~mm}$ [42]. In Wireless Networks-on-Chip, processing cores are connected to neighboring cores by electrical wires or optical interconnects, whereas distant cores communicate wirelessly to improve the latency and energy dissipation [43]. In the latter case, considering that up to 1000 processing cores could be integrated into a $300 \mathrm{~mm}^{2}$ die [44] and the reflections of the radiated waves within the wafer [45], typical transmission distances for wireless interconnects will be of several millimeters.

These values for the transmission distance, although low compared to current wireless communication systems, are around one order of magnitude larger than the wavelength of terahertz EM waves. As a consequence, graphennas will operate in far field conditions and macroscale physical channel models can be used to analyze GWC. The total attenuation, or path loss, in a GWC system can be expressed as [37]:

$$
A=A_{\text {spread }} A_{\text {abs }}
$$

where $A_{\text {spread }}$ is the spreading loss and $A_{a b s}$ is the molecular absorption loss, both expressed as multiplicative factors.

The molecular absorption loss, introduced in Sec. II, can be modeled by the following analytical expression [37]:

$$
A_{a b s}=\frac{1}{\tau}=e^{k(f) d}
$$

where $\tau$ is defined as the transmittance of the medium and $k$ is the medium absorption coefficient. Since we are interested in the scenario where the transmission distance tends to zero, we observe that in the limit $d \rightarrow 0$ the value of the molecular absorption loss tends to 1 :

$$
\lim _{d \rightarrow 0} A_{a b s}=\lim _{d \rightarrow 0} e^{k(f) d}=1
$$

Therefore, the molecular absorption loss will have a negligible effect in the envisaged scenarios of GWC. The total attenuation is then obtained considering only the spreading loss, given in the Fraunhofer region by the well-known expression for the free-space path loss:

$$
A=A_{\text {spread }}=\left(\frac{4 \pi f d}{c}\right)^{2}
$$

The antenna gain is expressed as a function of the effective area and the wavelength as $G=4 \pi A_{e f f} / \lambda^{2}$. Since, in a resonant patch antenna, both $A_{e f f}$ and $\lambda^{2}$ are proportional to the squared antenna length $\Delta^{2}$, the gain is a constant with respect to the scale parameters. Therefore, the values of the gain of the transmitting and receiving antennas will not influence our scalability analysis.

\section{Noise Power Spectral Density}

Finally, there are two main contributors to the ambient noise in the terahertz channel: thermal noise and molecular noise (introduced in Sec. II) [37]. The total noise power spectral density $N(f)$ can be calculated as the Boltzmann constant $k_{B}$ multiplied by the total noise temperature:

$$
N(f, d)=k_{B}\left(T_{\text {sys }}+T_{m o l}(f, d)\right)
$$

where $T_{\text {sys }}$ is the system temperature and $T_{m o l}$ is the molecular noise temperature. The molecular noise temperature can be expressed as follows [37]:

$$
T_{m o l}(f, d)=T_{0} \varepsilon=T_{0}(1-\tau)=T_{0}\left(1-e^{-k(f) d}\right)
$$

where $T_{0}$ is the standard temperature, $\varepsilon$ is known as the emissivity of the medium, $\tau$ is the transmittance of the medium and $k$ the medium absorption coefficient. As it happened with molecular absorption, molecular noise tends to disappear when the transmission distance is small:

$$
\lim _{d \rightarrow 0} T_{m o l}(f, d)=\lim _{d \rightarrow 0} T_{0}\left(1-e^{-k(f) d}\right)=0
$$

In consequence, the main contributor to the noise power spectral density in the terahertz channel at the nanoscale is the 
thermal noise. Approximating the system temperature by the standard temperature $T_{0}=293 \mathrm{~K}$, we obtain a constant noise power spectral density in the whole frequency band, denoted by $N_{0}$ :

$$
N(f, d)=k_{B} T_{\text {sys }}=k_{B} T_{0}=N_{0}
$$

Furthermore, there exist several recent efforts from the research community to provide models and experimental measurements of the noise in graphene devices [46]. The noise in graphene has been mainly characterized as flicker $(1 / f)$ noise for low frequencies $(1-10000 \mathrm{~Hz})$ [47] and shot noise for intermediate frequencies (hundreds of $\mathrm{MHz}$ ) [48]. Since terahertz wireless communications involve much higher frequencies, these noise components in graphene devices will not influence our performance analysis.

\section{Bandwidth}

As we have just observed, when the transmission distance is sufficiently small, molecular absorption and noise become negligible and the channel bandwidth is the whole terahertz band. Therefore, the total available bandwidth will be limited by the antenna bandwidth, which can be obtained as $B=f_{R} / Q$, where $f_{R}$ is the antenna resonant frequency and $Q$ its quality factor. The resonant frequency of metallic and graphene antennas, previously derived in Section II, can be expressed as:

$$
\begin{gathered}
f_{R m}=\frac{k_{1}^{\prime}}{\Delta} \\
f_{R g}=\frac{k_{2}^{\prime}}{\sqrt{\Delta}}
\end{gathered}
$$

where $k_{1}^{\prime}$ and $k_{2}^{\prime}$ are proportionality constants and $\Delta$ is the antenna length. We have observed in our previous work that the quality factor of metallic and graphene antennas does not significantly depend on the antenna size [8]. Therefore, we can define the new proportionality constants $k_{1}=k_{1}^{\prime} / Q$ and $k_{2}=k_{2}^{\prime} / Q$ to obtain an expression of the system bandwidth as a function of the scale parameters:

$$
\begin{gathered}
B_{m}=\frac{k_{1}}{\Delta} \\
B_{g}=\frac{k_{2}}{\sqrt{\Delta}}
\end{gathered}
$$

\section{E. Expression of the Channel Capacity}

Now, we combine the expressions obtained in (3), (7) and (11) with the definition of the channel capacity (2) in order to obtain the capacity of a nanoscale wireless communication channel:

$$
\begin{aligned}
C\left(B, d, P_{T}\right) & =\int_{0}^{B} \log _{2}\left(1+\frac{P_{T} / B}{\left(\frac{4 \pi f d}{c}\right)^{2} N_{0}}\right) d f \\
& =\frac{B}{\log 2} \log \left(1+\frac{c^{2} P_{T}}{(4 \pi d)^{2} B^{3} N_{0}}\right) \\
& +\frac{c \sqrt{P_{T}}}{2 \log (2) \pi d \sqrt{N_{0} B}} \arctan \frac{4 \pi d B^{3 / 2} \sqrt{N_{0}}}{c \sqrt{P_{T}}}
\end{aligned}
$$

Recalling the previous expressions for the bandwidth (14) and (15), we can express the channel capacity $C$ as a function of the three key scale parameters: the antenna length $\Delta$, the transmission distance $d$ and the transmitted power $P_{T}\left(c, N_{0}\right.$, $k_{1}$ and $k_{2}$ are constants):

$$
\begin{aligned}
C_{m}\left(\Delta, d, P_{T}\right) & =\frac{k_{1}}{\log (2) \Delta} \log \left(1+\frac{c^{2} \Delta^{3} P_{T} / d^{2}}{(4 \pi)^{2} N_{0} k_{1}^{3}}\right) \\
& +\frac{c \sqrt{\Delta P_{T} / d^{2}}}{2 \log (2) \pi \sqrt{N_{0} k_{1}}} \arctan \frac{4 \pi \sqrt{N_{0} k_{1}^{3}}}{c \sqrt{\Delta^{3} P_{T} / d^{2}}}
\end{aligned}
$$

$$
\begin{aligned}
C_{g}\left(\Delta, d, P_{T}\right) & =\frac{k_{2}}{\log (2) \sqrt{\Delta}} \log \left(1+\frac{c^{2} \Delta^{3 / 2} P_{T} / d^{2}}{(4 \pi)^{2} N_{0} k_{2}^{3}}\right) \\
& +\frac{c \sqrt[4]{\Delta} \sqrt{P_{T} / d^{2}}}{2 \log (2) \pi \sqrt{N_{0} k_{2}}} \arctan \frac{4 \pi \sqrt{N_{0} k_{2}^{3}}}{c \Delta^{3 / 4} \sqrt{P_{T} / d^{2}}}
\end{aligned}
$$

where we have isolated the factor $P_{T} / d^{2}$ in both expressions. We will see the capital importance of this quotient on the scalability of the channel capacity to the nanoscale in Sec. III.

\section{F. Quantitative Results}

In order to obtain quantitative results of the channel capacity as a function of $\Delta$ and $d$, we assign realistic values to the parameters of equations (17) and (18), considering an envisaged scenario of GWC. Next, we describe these values and the rationale behind the choices made.

First, the values of the constants $k_{1}^{\prime}$ and $k_{2}^{\prime}$ are obtained from our previous work in the antenna resonant frequency [35] as $k_{1}^{\prime}=1.7 \cdot 10^{8}$ and $k_{2}^{\prime}=4 \cdot 10^{9}$. We consider unity gain antennas and a quality factor $Q=5$, consistent with our previous work [35]. In consequence, the proportionality constants will be $k_{1}=3.4 \cdot 10^{7}$ and $k_{2}^{\prime}=8 \cdot 10^{8}$.

Regarding the transmitted signal, we consider a power spectral density of $10^{-18} \mathrm{~W} / \mathrm{Hz}$ over a bandwidth of $1 \mathrm{THz}$. We have chosen this value in light of recent results of the transmitted power by graphene antennas fed by a photoconductive source [49], whose radiated total power radiated is estimated to be in the $\mu \mathrm{W}$ range. Integrating this spectral density over a bandwidth of $1 \mathrm{THz}$ yields a total power of $1 \mu \mathrm{W}$, in agreement with the state of the art.

With respect to molecular absorption and noise, the medium absorption coefficient of a standard atmosphere has been found to have a maximum value of approximately $k_{\max }=$ 
TABLE II

VALUES CHOSEN FOR THE PARAMETERS

\begin{tabular}{cll}
\hline \hline Symbol & Quantity & Value \\
\hline$\Delta$ & Antenna length & $0.05-5 \mu \mathrm{m}$ \\
$d$ & Transmission distance & $1-10 \mathrm{~mm}$ \\
$P_{T}$ & $\begin{array}{l}\text { Average power radiated by the transmit- } \\
\text { ter }\end{array}$ & $1 \mu \mathrm{W}$ \\
& Proportionality constant in metallic an- & $3.4 \cdot 10^{7}$ \\
$k_{1}$ & tennas & $8 \cdot 10^{8}$ \\
$k_{2}$ & Proportionality constant in graphennas & $1.38 \cdot 10^{-23} \mathrm{~J} / \mathrm{K}$ \\
$k_{B}$ & Boltzmann constant & $293 \mathrm{~K}$ \\
$T_{0}$ & Standard temperature &
\end{tabular}

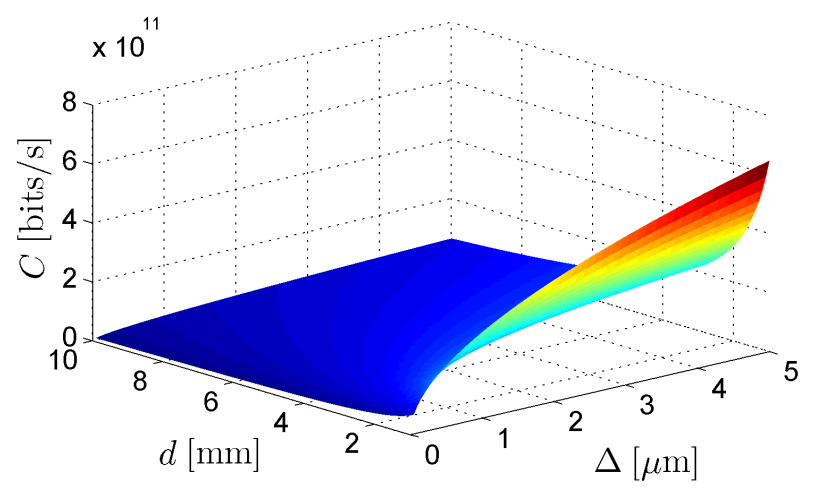

Fig. 3. Quantitative values of the channel capacity $C$ as a function of the antenna length $\Delta$ and the transmission distance $d$, when a metallic antenna is considered.

$3.5 \mathrm{~m}^{-1}$ [37]. Then, considering typical transmission distances for $\mathrm{GWC}$ of up to $d_{\max }=1 \mathrm{~cm}$, the maximum value of molecular absorption in a realistic GWC scenario is $A_{a b s}=e^{k_{\max } d_{\max }} \approx 1.036$, which is negligible in comparison with the attenuation due to the much larger spreading loss. This result confirms that molecular absorption will not have a significant effect on communication in short-range wireless communications, as we had previously obtained analytically. Similarly, the contribution of molecular noise is also negligible in comparison with thermal noise: $T_{\text {mol }}=T_{0}\left(1-e^{-k_{\max } d_{\max }}\right) \approx 0.034 T_{0}$. With respect to thermal noise, its spectral density has a value of $N_{0}=k_{B} T_{0} \approx$ $1.38 \cdot 10^{-23} \cdot 293 \mathrm{~W} / \mathrm{Hz}$.

Finally, we select a realistic range of values for the antenna length $\Delta$ and the transmission distance $d$. For the former, we choose the interval between 0.05 and $5 \mu \mathrm{m}$, and from 1 to $10 \mathrm{~mm}$ for the latter. In both cases, the chosen values are inspired by the range of envisaged values for nanosystems. In particular, since the free-space wavelength of terahertz waves is around $0.3 \mathrm{~mm}$, the far field assumption requires that the transmission distance is above a few millimeters.

A summary of the selected parameters is shown in Table II. The resulting quantitative values for the channel capacity are shown in Fig. 3 for a metallic antenna, and in Fig. 4 for a graphenna.

The results obtained when evaluating equations (17) and (18) show that the behavior of the channel capacity at the nanoscale differs significantly depending on whether we consider the use of graphennas in our analysis. We observe that,

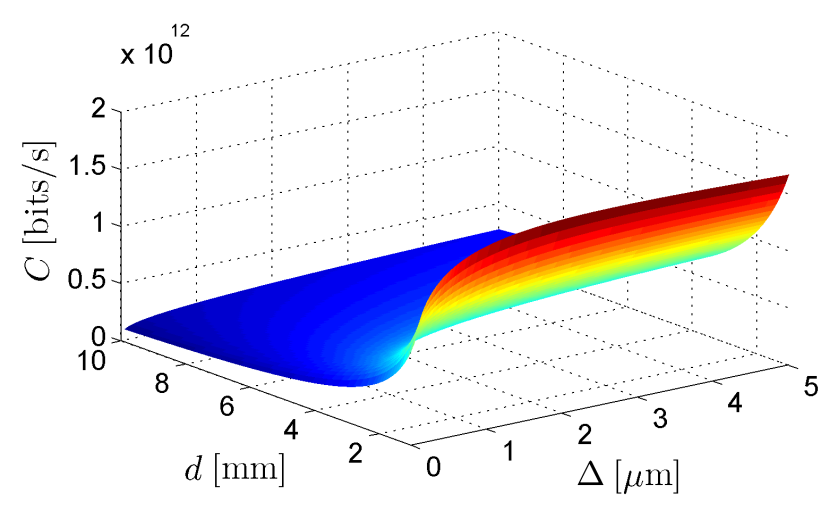

Fig. 4. Quantitative values of the channel capacity $C$ as a function of the antenna length $\Delta$ and the transmission distance $d$, when a graphenna is considered.

on the one hand, the absolute value of the channel capacity is up to twice as high when graphennas are used, with respect to metallic antennas. On the other hand, the channel capacity in graphennas decreases more slowly as their size $\Delta$ is reduced. This novel observation indicates that, thanks to the unique properties of plasmonic EM waves in graphene, the channel capacity in GWC scales better as the dimensions shrink than what is observed when conventional metallic antennas are considered.

The dependence of additional performance metrics of the graphene antenna has been studied in our previous work [8], finding that the antenna properties mainly depend on the antenna length, whereas their dependence on its width is small. Also the material and geometry of the dielectric substrate have a considerable influence, namely, a dielectric with low permittivity and a thickness equal to the resonant wavelength maximizes the antenna radiation efficiency. Furthermore, applying a chemical potential to the graphene patch will increase the antenna resonant frequency. Moreover, locating the antenna near the edge of the receiver yields a slightly better radiation efficiency, and the radiation pattern (and hence the directionality) of graphennas is not influenced by the antenna dimensions [50].

Finally, note that these results assume that the transmitted power has a constant value, which might not be realistic in some scenarios. For this reason, we next take an analytical approach and compute the limits of the channel capacity of wireless communications when the values of the three considered scale parameters, $\Delta, d$ and $P_{T}$, all tend to zero.

\section{LiMITS OF THE CHANNEL CAPACITY}

We are interested in finding closed-form expressions for the channel capacity given by (17) and (18) in the limit when all three scale parameters tend to zero, i.e., $\Delta \rightarrow 0$, $d \rightarrow 0$ and $P_{T} \rightarrow 0$. In this context, it is worth noting that the radiation properties of graphene nanoribbon antennas have shown that new effects need to be taken into account when calculating the electrical conductivity of thin graphene nanoribbons, due to the lateral confinement of electrons in such structures [51]. However, the scaling relationship between 
the antenna resonant frequency and its length is not affected. Therefore, our analysis remains valid also in the case of graphene nanoribbon antennas.

Unfortunately, the previously mentioned limits are not unique, but they depend upon the relationships among $\Delta$, $d$ and $P_{T}$. For instance, it can be seen intuitively that the channel capacity will increase if the transmission distance is reduced more rapidly (due to the reduced channel attenuation), while keeping the other scale parameters unchanged. Thus, in order to find an analytical expression for the capacity of a nanoscale wireless communications channel, we need to assume a given relationship among the scale parameters. Following an approach as general as possible, we express $d$ and $P_{T}$ as a function of $\Delta$ as follows:

$$
\begin{gathered}
d=k_{3} \Delta^{\alpha} \\
P_{T}=k_{4} \Delta^{\beta}
\end{gathered}
$$

where $k_{3}$ and $k_{4}$ are constants and $\alpha$ and $\beta$ are real positive exponents. In other words, we assume that when the antenna size $\Delta$ decreases, both the transmission distance $d$ and the transmitted power $P_{T}$ shrink as well, at relative rates $\alpha$ and $\beta$, respectively. Under these assumptions, we can express the channel capacity as a function of a single scale parameter, in this case $\Delta$, obtaining the following expressions:

$$
\begin{aligned}
C_{m}(\Delta) & =\frac{k_{11}}{\Delta} \log \left(1+k_{12} \Delta^{\beta-2 \alpha+3}\right) \\
& +k_{13} \Delta^{\frac{\beta-2 \alpha+1}{2}} \arctan \left(k_{14} \Delta^{-\frac{\beta-2 \alpha+3}{2}}\right) \\
C_{g}(\Delta)= & \frac{k_{21}}{\sqrt{\Delta}} \log \left(1+k_{22} \Delta^{\beta-2 \alpha+\frac{3}{2}}\right) \\
+ & k_{23} \Delta^{\frac{\beta-2 \alpha+1 / 2}{2}} \arctan \left(k_{24} \Delta^{-\frac{\beta-2 \alpha+3 / 2}{2}}\right)
\end{aligned}
$$

where $k_{i j}, i \in[1,2], j \in[1,4]$ are constants w.r.t. $\Delta$. The limits when $\Delta \rightarrow 0$ of the previous expressions are functions of the parameters $\alpha$ and $\beta$ :

$$
\begin{aligned}
& \lim _{\Delta \rightarrow 0} C_{m}(\Delta)= \begin{cases}\infty & \text { if } \beta-2 \alpha>-1, \\
\text { constant } & \text { if } \beta-2 \alpha=-1, \\
0 & \text { if } \beta-2 \alpha<-1 .\end{cases} \\
& \lim _{\Delta \rightarrow 0} C_{g}(\Delta)= \begin{cases}\infty & \text { if } \beta-2 \alpha>-1 / 2, \\
\text { constant } & \text { if } \beta-2 \alpha=-1 / 2, \\
0 & \text { if } \beta-2 \alpha<-1 / 2 .\end{cases}
\end{aligned}
$$

where the term $\beta-2 \alpha$ in the expressions above stems from the quotient $P_{T} / d^{2}=\Theta\left(\Delta^{\beta-2 \alpha}\right)$ that appears repeatedly in the capacity expressions (17) and (18).

We define the feasible region of the network as the scenario in which the channel capacity does not tend to zero when the network shrinks, i.e., the conditions under which $\lim _{\Delta \rightarrow 0} C(\Delta)>0$. When we compare the feasible regions in the cases of metallic antennas and graphennas, we identify

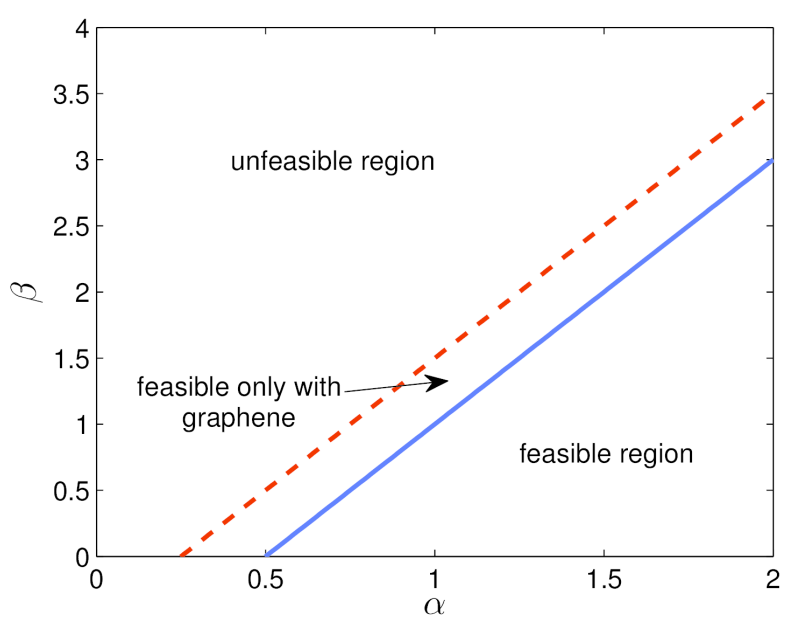

Fig. 5. Feasible area of the channel capacity as a function of $\alpha$ and $\beta$. The blue solid line corresponds to the case in which a metallic antenna is considered, and the red dashed line to a graphenna.

three clearly differentiated regions as a function of $\alpha$ and $\beta$, as shown in Fig. 5:

- The region $\beta-2 \alpha \geq-1 / 2$ is feasible both when metallic antennas and graphennas are used.

- The region $-1 \leq \beta-2 \alpha<-1 / 2$ is feasible only when graphennas are used.

- The region $\beta-2 \alpha<-1$ is never feasible.

We thus conclude that: (i) the quotient $P_{T} / d^{2}$ will be a key parameter in the scalability of GWC, since it will determine the feasibility of the network, (ii) the unique propagation properties of plasmonic EM waves in graphennas yield a larger feasible region as compared to when metallic antennas are used, and (iii) this scalability advantage of graphennas allows a reduction of the quotient $P_{T} / d^{2}$ up to a factor $\Theta\left(\Delta^{1 / 2}\right)$ with respect to metallic antennas as the network dimensions shrink.

\section{ScAlability GUiDELINES}

Several enormous challenges need to be faced by the scientific community when designing nanonetworks. First, as it occurs in current wireless networks, power consumption is envisaged to become a bottleneck in the performance of nanonetworks. Hence, one of these challenges is the fabrication of nanoscale power sources able to provide energy to nanosystems. In this direction, researchers have started working on novel nanomaterials and techniques to create nanobatteries [52], as well as nanoscale energy-harvesting modules [53].

Besides the power source, another important challenge of nanonetworks is the expectedly large density of nanosystems required to cover a certain region. Indeed, because of the very limited transmission range of nanosystems, and since the number of nodes $N$ needed to cover a fixed 3-dimensional space scales with their transmission range as $N=\Theta\left(d^{-3}\right)$, it seems likely that a huge number of nodes will be required to build a nanonetwork. The cost of manufacturing such a large 


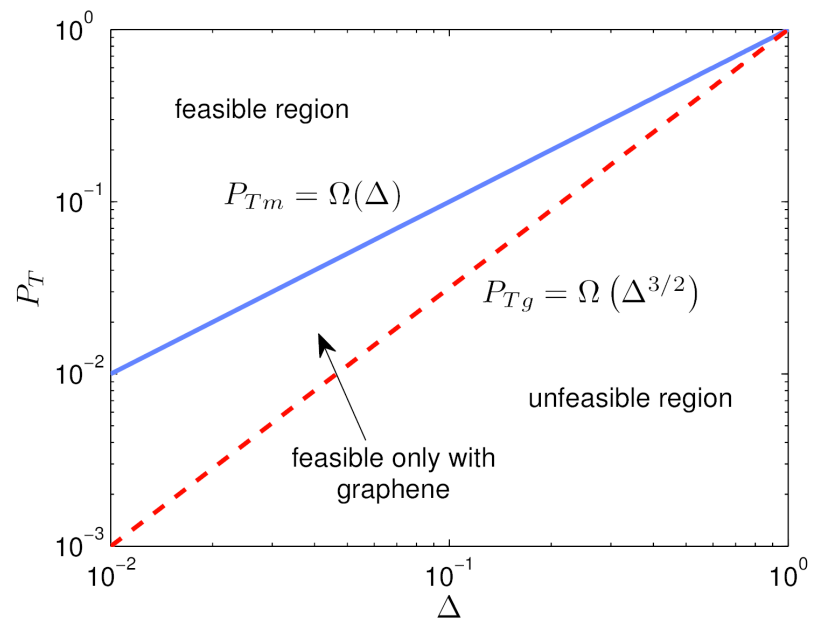

Fig. 6. Log-log plot comparing the scalability of the transmitted power by a metallic antenna $\left(P_{T_{m}}\right.$, blue solid line), compared to a graphenna $\left(P_{T_{g}}\right.$, red dashed line), as a function of the antenna length $\Delta$. The transmission distance scales as $d=\Theta(\Delta)$.

quantity of nanosystems may compromise the feasibility of the nanonetwork.

With this concern in mind, we establish an additional condition for the network feasibility. In addition to the constraint introduced in the previous section that the channel capacity does not tend to zero when the network shrinks, i.e., $\lim _{\Delta \rightarrow 0} C(\Delta)>0$, we restrict the total number of nanosystems $N$ to be inversely proportional to the volume of a single nanosystem $V$, i.e., $N \propto 1 / V$. The rationale behind this choice is that, in this scenario, the combined volume of all the nanosystems $N V$ (and thus their cost) will be constant. Since the volume of a nanosystem scales as $V=\Theta\left(\Delta^{3}\right)$, this condition results in $\Theta\left(d^{-3}\right)=\Theta\left(\Delta^{-3}\right)$. From this expression, we derive that the necessary condition for the network feasibility is $d=\Theta(\Delta)$. In other words, all the network dimensions, i.e., the nanosystem size and the transmission distance, must shrink proportionally. Note that the same condition would be obtained assuming a 2dimensional scenario, where the number of nanosystems is inversely proportional to the area of a single nanosystem.

In this scenario, which corresponds to $\alpha=1$, the feasible region of the network corresponds to the values $\beta \leq 1$ when a metallic antenna is considered, and $\beta \leq 3 / 2$ with a graphenna. In other words, with a metallic antenna, the transmitted power needs to scale at most linearly with the antenna size, as $P_{T_{m}}=\Omega(\Delta)$; however, graphennas allow a faster decrease in the transmitted power, at a rate $P_{T_{m}}=\Omega\left(\Delta^{3 / 2}\right)$. Both cases are compared in Fig. 6. As a consequence, graphennas achieve a scaling advantage of $\Theta\left(\Delta^{1 / 2}\right)$ with respect to metallic antennas. We extract the following scalability guideline: the use of graphennas allows relaxing the requirements for nanobatteries and nano-energy harvesting modules that will power nanosystems; for instance, when the antenna size is reduced by two orders of magnitude, the required energy is one order of magnitude smaller when using graphennas instead of metallic antennas.

\section{Conclusions}

In this paper, we analyze the scalability trends of the channel capacity in wireless communications to the nanoscale, which may be enabled by graphene plasmonic miniaturized antennas, or graphennas. The propagation of EM waves in graphennas exhibits novel plasmonic effects that will affect their communication properties. These effects do not appear when conventional metallic antennas are simply reduced in size.

We identify and analyze the impact of three of these novel properties: the propagation of plasmonic waves in graphennas, molecular absorption and molecular noise. Our results show that, while the influence of the two latter effects tends to disappear as the transmission distance shrinks, the unique plasmonic properties of graphennas have a great impact on the channel capacity in GWC. We evaluate this impact both graphically, by plotting quantitative values of the channel capacity as a function of the antenna length and the transmission distance, and analytically, by computing the limits on the channel capacity when the network size tends to zero. In both cases, we find the channel capacity to scale better as the dimensions shrink when graphennas are used with respect to the case of metallic antennas. By deriving conditions to ensure the feasibility of nanonetworks, we quantify the scalability advantage derived from the use of graphennas as a function of the transmitted power and the transmission distance.

Finally, we derive guidelines which indicate how network parameters need to scale in order to keep the network feasible. We find two necessary conditions for the network feasibility: (i) the transmission distance needs to scale as $\Theta(\Delta)$, and (ii) the transmitted power needs to scale as $\Omega(\Delta)$ when metallic antennas are used, and as $\Omega\left(\Delta^{3 / 2}\right)$ when graphennas are used. Therefore, graphennas present a scalability advantage in the transmitted power of $\Theta\left(\Delta^{1 / 2}\right)$. In other words, when the antenna length is reduced by two orders of magnitude, the required energy is one order of magnitude smaller when using graphennas with respect to the metallic case.

These results establish a general framework which may serve designers as a guide to implement the future wireless communication networks among nanosystems.

\section{ACKNOWLEDGMENT}

This work has been partially supported by the FPU grant of the Spanish Ministry of Education and the Comissionat per a Universitats i Recerca of the Catalan Government (Ref. 2014SGR-1427).

\section{REFERENCES}

[1] I. F. Akyildiz, F. Brunetti, and C. Blázquez, "Nanonetworks: A new communication paradigm," Computer Networks, vol. 52, no. 12, pp. 2260-2279, 2008

[2] I. F. Akyildiz and J. M. Jornet, "Electromagnetic wireless nanosensor networks," Nano Communication Networks, vol. 1, no. 1, pp. 3-19, 2010.

[3] S. Abadal, E. Alarcón, M. C. Lemme, M. Nemirovsky, and A. CabellosAparicio, "Graphene-enabled Wireless Communication for Massive Multicore Architectures," IEEE Communications Magazine, vol. 51, no. 11, pp. 137-143, 2012.

[4] I. F. Akyildiz and J. M. Jornet, "The Internet of nano-things," IEEE Wireless Communications, vol. 17, no. 6, pp. 58-63, 2010. 
[5] J.-H. Park, G. von Maltzahn, L. L. Ong, A. Centrone, T. A. Hatton, E. Ruoslahti, S. N. Bhatia, and M. J. Sailor, "Cooperative nanoparticles for tumor detection and photothermally triggered drug delivery," Advanced Materials, vol. 22, no. 8, pp. 880-885, 2010.

[6] J. Han, J. Fu, and R. B. Schoch, "Molecular sieving using nanofilters: past, present and future," Lab on a chip, vol. 8, no. 1, pp. 23-33, 2008.

[7] J. M. Jornet and I. F. Akyildiz, "Graphene-Based Nano-Antennas for Electromagnetic Nanocommunications in the Terahertz Band," in European Conference on Antennas and Propagation, Barcelona, 2010.

[8] I. Llatser, C. Kremers, A. Cabellos-Aparicio, J. M. Jornet, E. Alarcón, and D. N. Chigrin, "Graphene-based nano-patch antenna for terahertz radiation," Photonics and Nanostructures - Fundamentals and Applications, vol. 10, no. 4, pp. 353-358, 2012.

[9] K. Novoselov, A. Geim, S. Morozov, D. Jiang, Y. Zhang, S. V. Dubonos, I. V. Grigorieva, and A. A. Firsov, "Electric field effect in atomically thin carbon films." Science, vol. 306, no. 5696, pp. 666-9, 2004.

[10] I. Llatser, C. Kremers, A. Cabellos-Aparicio, J. M. Jornet, E. Alarcón, and D. N. Chigrin, "Scattering of terahertz radiation on a graphene-based nano-antenna," AIP Conference Proceedings, vol. 1398, pp. 144-146, 2011.

[11] M. Tamagnone, J.-S. Gómez-Díaz, J. R. Mosig, and J. PerruisseauCarrier, "Analysis and design of terahertz antennas based on plasmonic resonant graphene sheets," Journal of Applied Physics, vol. 112, no. 11, p. 114915, 2012.

[12] L. Ju, B. Geng, J. Horng, C. Girit, M. Martin, Z. Hao, H. a. Bechtel, X. Liang, A. Zettl, Y. R. Shen, and F. Wang, "Graphene plasmonics for tunable terahertz metamaterials," Nature Nanotechnology, vol. 6, no. 10, pp. 630-634, 2011.

[13] H. Yan, X. Li, B. Chandra, G. Tulevski, Y. Wu, M. Freitag, W. Zhu, P. Avouris, and F. Xia, "Tunable infrared plasmonic devices using graphene/insulator stacks," Nature Nanotechnology, vol. 7, no. 5, pp. 330-334, 2012.

[14] I. Crassee, M. Orlita, M. Potemski, A. Walter, M. Ostler, T. Seyller, I. Gaponenko, J. Chen, and A. Kuzmenko, "Intrinsic terahertz plasmons and magnetoplasmons in large scale monolayer graphene," Nano letters, vol. 12, no. 5, pp. 2470-2474, 2012.

[15] A. Y. Nikitin, F. Guinea, F. J. Garcia-Vidal, and L. Martin-Moreno, "Surface plasmon enhanced absorption and suppressed transmission in periodic arrays of graphene ribbons," Physical Review B, vol. 85, no. 8, p. 081405, 2012.

[16] M. C. Lemme, T. J. Echtermeyer, M. Baus, and H. Kurz, "A Graphene Field-Effect Device," IEEE Electron Device Letters, vol. 28, no. 4, pp. 282-284, 2007.

[17] L. Liao, Y.-C. Lin, M. Bao, R. Cheng, J. Bai, Y. Liu, Y. Qu, K. L. Wang, Y. Huang, and X. Duan, "High-speed graphene transistors with a self-aligned nanowire gate," Nature, vol. 467, no. 7313, pp. 305-308, 2010.

[18] Y. Wu, Y.-m. Lin, A. A. Bol, K. A. Jenkins, F. Xia, D. B. Farmer, Y. Zhu, and P. Avouris, "High-frequency, scaled graphene transistors on diamond-like carbon," Nature, vol. 472, no. 7341, pp. 74-78, 2011.

[19] H. Wang, A. Hsu, J. Wu, J. Kong, and T. Palacios, "Graphene-based ambipolar RF mixers," IEEE Electron Device Letters, vol. 31, no. 9, pp. 906-908, 2010

[20] H. Wang, A. Hsu, K. K. Kim, J. Kong, and T. Palacios, "Gigahertz ambipolar frequency multiplier based on cvd graphene," in IEEE International Electron Devices Meeting, 2010, pp. 23.6.1 -23.6.4.

[21] T. Palacios, A. Hsu, and H. Wang, "Applications of graphene devices in RF communications," IEEE Communications Magazine, vol. 48, no. 6 , pp. 122-128, 2010.

[22] Y.-M. Lin, A. Valdes-Garcia, S.-J. Han, D. B. Farmer, I. Meric, Y. Sun, Y. Wu, C. Dimitrakopoulos, A. Grill, P. Avouris, and K. a. Jenkins, "Wafer-scale graphene integrated circuit." Science, vol. 332, no. 6035, pp. 1294-7, 2011.

[23] P. Avouris, Y. Wu, and W. Zhu, "Graphene devices and semiconductor field effect transistors in 3D hybrid integrated circuits," 2012, US Patent App. 13/352,737.

[24] Y.-M. Lin and J.-B. Yau, "Metal-free integrated circuits comprising graphene and carbon nanotubes," 2012, US Patent 20,120,326,129.

[25] T. Mueller, F. Xia, and P. Avouris, "Graphene photodetectors for highspeed optical communications," Nature Photonics, vol. 4, no. 5, pp. 297-301, 2010

[26] B. Gulbahar and O. Akan, "A Communication Theoretical Modeling of Single-Layer Graphene Photodetectors and Efficient Multireceiver Diversity Combining," IEEE Trans. on Nanotechnology, vol. 11, no. 3, pp. 601-610, 2012
[27] R. Dennard, F. Gaensslen, V. Rideout, E. Bassous, and a.R. Leblanc, "Design Of Ion-implanted MOSFET's with Very Small Physical Dimensions," Proc. of the IEEE, vol. 87, no. 4, pp. 668-678, 1999.

[28] M. Wautelet, "Scaling laws in the macro- , micro- and nanoworlds," European Journal of Physics, vol. 22, no. 6, pp. 601-611, 2001.

[29] D. Foty, "Perspectives on scaling theory and CMOS technology understanding the past, present, and future," in IEEE International Conference on Electronics, Circuits and Systems, 2004, pp. 631-637.

[30] P. Gupta and P. R. Kumar, "The capacity of wireless networks," IEEE Trans. on Information Theory, vol. 46, no. 2, pp. 388-404, 2000.

[31] O. Lévêque and I. E. Telatar, "Information-Theoretic Upper Bounds on the Capacity of Large Extended Ad Hoc Wireless Networks," IEEE Trans. on Information Theory, vol. 51, no. 3, pp. 858-865, 2005.

[32] J. Ahn and B. Krishnamachari, "Fundamental Scaling Laws for EnergyEfficient Storage," in ACM MobiHoc, 2006, pp. 334-343.

[33] A. Vakil and N. Engheta, "Transformation optics using graphene," Science, vol. 332, no. 6035, pp. 1291-4, 2011.

[34] M. Jablan, H. Buljan, and M. Soljačić, "Plasmonics in graphene at infrared frequencies," Physical Review B, vol. 80, no. 24, 2009.

[35] I. Llatser, C. Kremers, A. Cabellos-Aparicio, E. Alarcón, and D. N. Chigrin, "Comparison of the resonant frequency in graphene and metallic nano-antennas," in AIP Conference Proceedings, vol. 143, 2012, pp. 143-145.

[36] R. Piesiewicz, T. Kleine-Ostmann, N. Krumbholz, D. Mittleman, M. Koch, J. Schoebei, and T. Kurner, "Short-Range Ultra-Broadband Terahertz Communications: Concepts and Perspectives," IEEE Antennas and Propagation Magazine, vol. 49, no. 6, pp. 24-39, 2007.

[37] J. M. Jornet and I. F. Akyildiz, "Channel Modeling and Capacity Analysis for Electromagnetic Wireless Nanonetworks in the Terahertz Band," IEEE Trans. on Wireless Communications, vol. 10, no. 10, pp. 3211-3221, 2011

[38] C. E. Shannon, "The mathematical theory of communication. 1963." The Bell System Technical Journal, vol. 27, pp. 379-423, 623-656, 1948.

[39] A. Goldsmith, Wireless Communications. Cambridge Univ. Press, 2005.

[40] S. Verdu, "Spectral efficiency in the wideband regime," IEEE Trans. on Information Theory, vol. 48, no. 6, pp. 1319-1343, 2002.

[41] J. M. Jornet and I. F. Akyildiz, "Information Capacity of Pulse-based Wireless Nanosensor Networks," in IEEE SECON, Salt Lake City, 2011.

[42] M. Pierobon, J. M. Jornet, N. Akkari, S. Almasri, and I. F. Akyildiz, "A routing framework for energy harvesting wireless nanosensor networks in the THz band," Wireless Networks, vol. 20, no. 5, pp. 1169-1183, 2014.

[43] A. Ganguly, K. Chang, S. Deb, P. Pande, B. Belzer, and C. Teuscher, "Scalable hybrid wireless network-on-chip architectures for multicore systems," IEEE Trans. on Computers, vol. 60, no. 10, pp. 1485-1502, 2011.

[44] S. Borkar, "Thousand core chips: A technology perspective," in Proceedings of the Annual Design Automation Conference. New York: ACM, 2007, pp. 746-749.

[45] M. Sun, Y. P. Zhang, G. X. Zheng, and W.-Y. Yin, "Performance of intrachip wireless interconnect using on-chip antennas and UWB radios," IEEE Trans. on Antennas and Propagation, vol. 57, no. 9, pp. 27562762, 2009.

[46] M. Andersson, "Noise characterisation of graphene FETs," Master's thesis, Chalmers University of Technology, 2012.

[47] G. Liu, W. Stillman, S. Rumyantsev, Q. Shao, M. Shur, and A. Balandin, "Low-frequency electronic noise in the double-gate single-layer graphene transistors," Applied Physics Letters, vol. 95, no. 3, 2009.

[48] R. Danneau, F. Wu, M. Craciun, S. Russo, M. Tomi, J. Salmilehto, A. Morpurgo, and P. Hakonen, "Shot noise in ballistic graphene," Physical review letters, vol. 100, no. 19, p. 196802, 2008.

[49] A. Cabellos-Aparicio, I. Llatser, E. Alarcón, A. Hsu, and T. Palacios, "Use of $\mathrm{THz}$ photoconductive sources to characterize graphene RF plasmonic antennas," arXiv preprint arXiv:1401.6878, 2014.

[50] I. Llatser, C. Kremers, D. N. Chigrin, J. M. Jornet, M. C. Lemme, A. Cabellos-Aparicio, and E. Alarcón, "Characterization of Graphenebased Nano-antennas in the Terahertz Band," in European Conference on Antennas and Propagation, 2012.

[51] J. M. Jornet and I. F. Akyildiz, "Graphene-based Plasmonic Nanoantenna for Terahertz Band Communication in Nanonetworks," IEEE JSAC, vol. 31, no. 12, pp. 685 - 694, 2013.

[52] F. Vullum and D. Teeters, "Investigation of lithium battery nanoelectrode arrays and their component nanobatteries," Journal of Power Sources, vol. 146, no. 1-2, pp. 804-808, 2005.

[53] X. Wang, L. Zhi, and K. Müllen, "Transparent, conductive graphene electrodes for dye-sensitized solar cells," Nano letters, vol. 8, no. 1, pp. 323-7, 2008. 